\title{
Fuzzy Multicriteria analysis for evaluation of risks in complex engineering projects in the Aeronautical industry
}

\author{
H. Fukayama ${ }^{1}$, E. Fernandes ${ }^{2}$ \& N. F. F. Ebecken ${ }^{1}$ \\ ${ }^{I}$ Department of Civil Engineering, \\ Federal University of Rio de Janeiro, Brazil \\ ${ }^{2}$ Department of Production Engineering, \\ Federal University of Rio de Janeiro, Brazil
}

\begin{abstract}
This paper presents an application, in the aeronautical industry, of a risk management methodology based on Fuzzy Multicriteria analysis (FMA). The proposed methodology analyses external and internal risks factors, formulating indicators that can be evaluated in some levels of the decision-making process (operational, tactical and strategic). The results show a way to obtain a more holistic vision of the risk in projects, assisting managers in the coordination of all possible risk factors that can affect the final result. Moreover, the results also demonstrate great chances of profits in cost, because of the faster analysis and the possibility to use in preliminary phases of the project.
\end{abstract}

Keywords: risk management, engineering projects management, Fuzzy Multicriteria analysis.

\section{Introduction}

The management of projects happens in an environment more dynamic and complex each day and that constantly, it is confronted with several risks and uncertainties. This way, it is imperative that the managers dispose of agile mechanisms of risk evaluation so that they can consider all the possible factors that can affect the final result of a project. An effective approach of risk management makes available a tool for the engineering project manager to identify and to evaluate potential risks quickly and precision. The studies of PMI [1] in project risk management show that the best practices observed, may be 
done with analyses of external and internal aspects. This work aims to improve these analyses through the decision-making process based on Fuzzy Multicriteria analysis (FMA). Pender [2] has already indicated the use of FMA for risk evaluation in projects. The proposed methodology presents a systematic that allows the manager to evaluate project risk factors in the several dimensions of the decision-making process, taking as references strips of risks in function of the specialist's conservative or aggressive positioning. The expert's attitude will depend on the weight and risk criteria determined in the evaluation. The dynamics of business environments have been demanding substantial flexibility in the process of Project Management; moreover, these environments become more complex each day. This complex environment results from a series of external and internal factors that have been modified in projects. Williams [3] already observed that, due to projects become more complex each day, the traditional methods of project management were not shown appropriate anymore, requiring new methods for analysis and management.

In this dynamic and complex context, it is increasing the number of propositions and discussions on risks in engineering projects. This work makes a brief revision of the literature on Risk management in complex projects and in Fuzzy Multicriteria analysis (FMA). After that, it is proposal an analytical methodology for risk evaluation in complex projects of engineering, involving the concepts of FMA.

\section{Revision of the literature of risk in complex projects}

It is not intended here to revise the whole literature on management of project risk, but to select papers related to the risks in complex engineering projects. Kangari and Riggs [4] alerted that most of the risk factors in projects are not always possible to transform in numerical data, in this cases, they suggest the linguistic approach. Antonsson and Otto [5] developed a method based on fuzzy logic to manage the uncertainties in engineering projects. This method called MoI (Method of Imprecision) is applied in the preliminary phases of projects developments, where the "imprecision" and the uncertainty are higher. Ayyub et al. [6] makes an approach of Risk Management based on fuzzy logic for the development of a four-stroke diesel engine ship. Due to the analyses in preliminaries phases of the projects are based on subjective information and opinions from specialists, one of the conclusions of this study was the confirmation of the fuzzy methodology as an appropriate technique to be applied in these steps. Ayyub et al. [6] also defines five risk categories: Cost and Equipment damage, Operability, Maintainability, Personnel Death/Injury and Environmental Impact. Mustafa and Al-Bahar [7] presented six categories for risk evaluation in construction projects: Acts of God risks or Natural phenomena (earthquakes, floods, fires, landslides, etc); Physical risks (damage to structure or property, damage to equipment and material, labor injuries and death, etc.); Financial and Economical risks (Inflation, Financings, Exchange rate, etc.); Political and Environmental risks (Changes in the legislation, Wars, Regulation, Pollution, etc.); Design risks (Scope, Specifications, Project Changes, etc.); and 
Operational Factors (Labor Disputes, Productivity, Equipments damages, etc.). It is not observed in the literature a significant alteration of these categories in more recent works. Some researches suggest the adaptation of risk factors to the projects. Akintoye and MacLeod [8] applied questionnaires to identify specific risk factors, which were observed by the workers of the construction industry. In 2001 several articles were published in International Journal of Project Management about risks in project. Two of them approach the use of the fuzzy logic in the evaluation of project risks (Pender [2] and Kuchta [9]) and two focused in great engineering projects (Miller and Lessard [10] and Floricel and Miller [11]). Charoenngam and Yeh [12] made an approach, well inside of the scope of the present work, to deal with risk factors in the construction of hydroelectric power stations. Moreover, they identified the categories of risks and classified in: Financial \& Economic, Political \& Environment, Design, Physical, Site Construction and Acts of God. These items are similar to the specifications mentioned before. By the presented bibliographical revision, it can be observed that the researches on risks, in several types of engineering projects, show the need for the development of a specific approach. Aiming to develop robust tools for risk management in complex projects, this work explores the use of FMA for risk evaluation.

\section{Analytical methodology}

The theory of fuzzy sets was introduced by Zadeh [13] as a generic approach to express the different types of uncertainty inherent in human systems. According to Zadeh [13], our ability to make precise and significant statements about systems behaviour has declined as they have become more complex. He proposed the use of fuzzy sets and approximation methods to model such systems. Ayyub et al. [6] concluded in his study that, for hazard assessment analysis, the softwares based on fuzzy logic are a simple and very flexible tool. The hazard analysis based on the fuzzy risk ranking better reflects some of the real scenarios of the system possible failures.

The main element that guides decision making in fuzzy modelling is a rule in the form: if A (observed event - input) then B (resulting event - output). The observed events and resulting events are expressed in linguistic terms (Figure 1 and Figure 2, respectively). These linguistic terms aim to represent the complexity of the measurement. The fuzzy set of inputs A (Figure 1) and outputs $\mathrm{B}$ (Figure 2) can be represented, respectively, by equations (1) and (2.)

$$
\begin{aligned}
& \mathrm{A}=\{x, f(x), x \in \mathfrak{R} \text { and } f(x) \in \mathfrak{R} \mid 1 \leq x \leq 9 \text { and } 0 \leq f(x) \leq 1\} \\
& \mathrm{B}=\{y, f(y), y \in \mathfrak{R} \text { and } f(y) \in \mathfrak{R} \mid 0 \leq y \leq 1 \text { and } 0 \leq f(y) \leq 1\}
\end{aligned}
$$

For example, a given input (Figure 1) can be measured in 3 categories, which are: Low risk (L), Medium risk (M) and High risk $(\mathrm{H})$. This is one way that people reason inherently, but if a specialist is asked to define, in a scale from 1 to 9 , the condition of a certain input, being 1 the best situation and 9 the worst, he may say the number 7.5. Considering the linguistic term in Figure 1, the specialist could be said to have indicated a state between medium and high, that is, comprising a medium classification component $(\mathrm{M})$ and a high component 


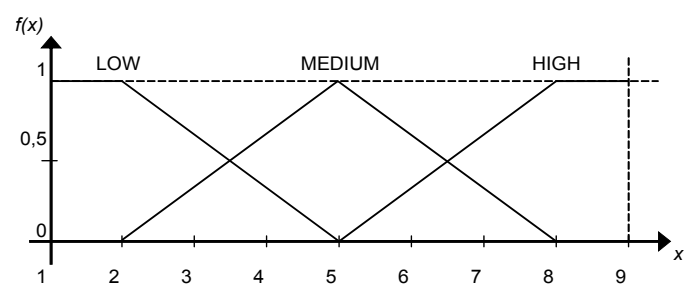

Figure 1: $\quad$ Linguistic term (input).

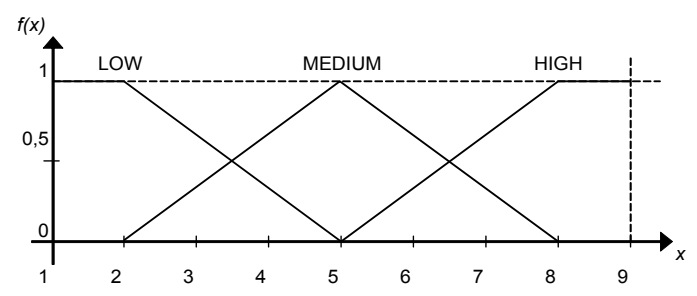

Figure 2: $\quad$ Linguistic term of the result indicators (output).

$(\mathrm{H})$, an attitude which is reflected on the $f(X)$ axis, where 0 (zero) represents no adhesion to the classification and 1 total adhesion to the classification. For instance, according to the linguistic term in Figure $1, f\left(X_{B}\right)$ and $f\left(X_{M}\right)$, according to the equations (3) and (4), being $X$ a real number varying from 1 to 9.

$$
\begin{aligned}
& f\left(X_{B}\right)= \begin{cases}1, & \text { if } X<2 \\
\frac{5-X}{3}, & \text { if } 2 \leq X \leq 5\end{cases} \\
& f\left(X_{M}\right)= \begin{cases}0, & \text { if } X>5 \\
\frac{X-2}{3}, & \text { if } X<2 \\
\frac{8-X}{3}, & \text { if } 5 \leq X \leq 8<5 \\
0, & \text { if } X>8\end{cases} \\
& f\left(X_{A}\right)= \begin{cases}0, & \text { if } X<5 \\
\frac{X-5}{3}, & \text { if } 5 \leq X \leq 8 \\
1, & \text { if } X>8\end{cases}
\end{aligned}
$$

Thus, to each classification there will be a corresponding mathematical expression to define $f(X)$ for a given evaluation. In this way, for $\mathrm{x}=7.5, f\left(X_{B}\right)$ will be equal 0 (zero), $f\left(X_{M}\right)$ will be equal 0.17 (zero point one seven) and $f\left(X_{A}\right)$ will be equal 0.83 (zero point eight three). Analogous reasoning can be applied to the outputs. The rules of the decision-making process (if $\mathrm{x}$ then $\mathrm{y}$ ) are subject to weightings $(W)$. This weighting reflects the relative influence of the rule in the result, because the result is composed of an operation of union of the rules 
according to a certain criterion. Given these elements, it is used tools of the set theory for the process called fuzzification and through a deterministic mathematical method called defuzzification is obtained the numeric result of the analysis. The result of this process supplies an index resulting from the application of the inputs in the unit of observation being monitored, according to the fuzzy model defined for the analysis [14]. Through this index, built by several inputs and in agreement with specialist opinions, is determined the level of project risk for each category of risk indicators.

Gheorghe et al. $[15,16]$ developed an approach that brings some flexibility to the precise manner in which specialists present their evaluations of criteria. Using the alpha-cut concept, they consider decision-maker's behaviours in two dimensions: one being their degree of aggressiveness (aggressive or conservative), another being their degree of optimism (optimistic or pessimistic). In this way Gheorghe et al. [16] developed an analysis where the lower limit $\left(W_{L B}\right)$ and the upper limit $\left(W_{U B}\right)$ of the criterion weightings define the decisionmaker's degree of aggressiveness and the lower limits $\left(C_{L B}\right)$ and upper limits $\left(C_{U B}\right)$ of the criterion evaluation define the decision-maker's degree of optimism. Likewise this wider concept of the result, the present work defines a surface of the FMA evaluation instead of a single point. Figure 3 shows the surface of solutions of the fuzzy analysis depending on the decision-maker's attitude, adapted from Gheorghe et al. [16]. We can verify five notable points in this surface: four extreme points defined by the combinations $W_{L B} C_{L B}, W_{L B} C_{U B}, W_{U B}$ $C_{L B}, W_{U B} C_{U B}$; and one moderate point defined by the measures $W_{M E} C_{M E}$.

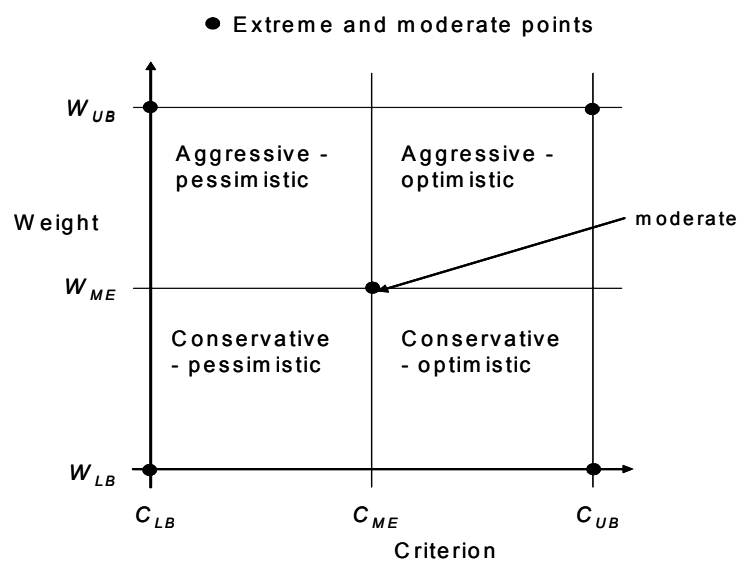

Figure 3: $\quad$ Surface of solution of the alpha-cut fuzzy analysis.

Considering the result indicator of project risk evaluation as a thermometer, it can be obtained a linear variation for it, in agreement with the decision-maker's attitude. Hence, it supplies a strip of result indicator in terms of lower and upper bounds. In this kind of analysis, the results are more realistic than that observed by medium point only. For the case study of this paper, the points $W_{L B} C_{L B}, W_{M E}$ $C_{M E}$ and $W_{U B} C_{U B}$ have been calculated, characterizing the lower, moderate and 
upper points of the risk indicators. The analytical methodology, developed here, has three fuzzy levels of risk indicators, as shown in the Figure 4. The primary indicators are those obtained through the questions answered by the specialists $\left(\mathrm{RI}_{111}\right.$ to $\left.\mathrm{RI}_{235}\right)$. From the primary indicators, the first analysis level can be developed the fuzzy risk indicators of operational level $\left(\mathrm{RI}_{11}\right.$ to $\left.\mathrm{RI}_{23}\right)$. The operational fuzzy indicators are thus aggregated at the tactical level, generating the fuzzy internal and external indicators $\left(\mathrm{RI}^{\mathrm{I}}\right.$ and $\left.\mathrm{RI}^{\mathrm{E}}\right)$. Eventually, these two indicators at the tactical level are aggregated into one fuzzy indicator at the strategic level (RI). This thus constitutes a coherent system of cause and effect, which makes possible to monitor the evolution of the risk parameters at the operational, tactical and strategic levels. This methodology, in addition of presenting a clear and transparent vision of how the managers perceive the question of project risk, also presents a simulator that allows the manager to evaluate the impact of improvements in certain indicators in the overall context of the risk analysis, this in the three levels of management: the operational, the tactical and the strategic.

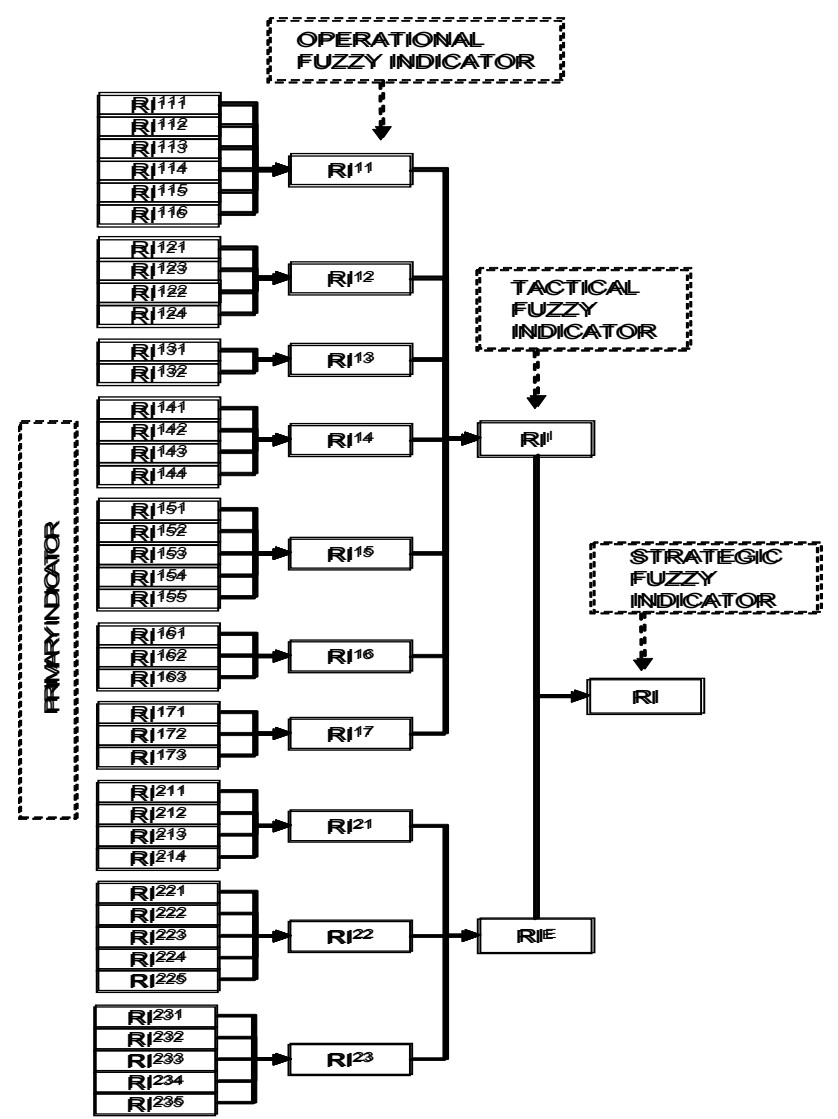

Figure 4: $\quad$ Project risk management structure. 


\section{Study of case}

To evaluate the proposed methodology, it was chosen the aeronautical industry due to some of its characteristics, which are important for this case study, such as high complexity and uncertainty. The project used in the analyses was the development of an aircraft for the Executive market (ultra-large category) with value about 40 million dollars. The foreseen duration of this project was esteemed initially in 2 years. In agreement with In agreement with Dinsmore et al. [17], in the beginning of the project there are more uncertainties involved that decreases as the project advances. The impact of the risks, unlike the uncertainty, increases as the time passes and the end of the project is going arriving. Figure 5, extracted from Dinsmore et al. [17], show in a generic way, the evolution of the uncertainties and the impact of the risk along the project life cycle.

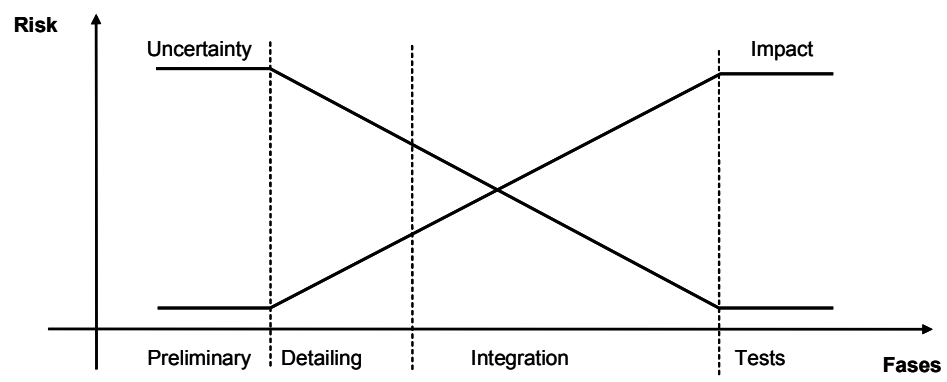

Figure 5: Uncertainty versus impact of the risk in the life cycle of the project.

With the objective of acquiring a better result in the application of the proposed methodology, it was chosen the Detailing Phase of the project for application of the questionnaire. This choice is due to existing in this phase the following items:

- Teams already formed and with the minimum knowledge necessary on the project;

- All of the technological areas (structures, electric, propulsion, aeronautics, etc) necessary for the development of the product already involved;

- Uncertainties due to the final result to be difficult to evaluate.

The applied questionnaire was structured in agreement with the following division: Internal factors (Scope, Programming, Budget, RH, Other, Technology and Performance) and External Factors (Financial \& Economical, Political \& Environmental and Actions of the Nature). The chosen division seeks to cover all the possible areas that may affect the final result of the project. The choice of this structure, as well as the formulated subjects (primary indicators) they are in agreement with several other questionnaires and/or methodologies used for the identification of risks in projects. Table 1 shows the Primary and Fuzzy Indicators of analyses.

In the fulfilling of the questionnaire participated 16 specialists, among them, the own Project Manager responsible for the development of this product. The 
Table 1: $\quad$ Primary and fuzzy indicators.

\begin{tabular}{|c|c|}
\hline$R^{1} I^{1}$ & INTERNAL FACTORS \\
\hline $\mathrm{RI}^{11}$ & SCOPE \\
\hline $\mathrm{RI}^{111}$ & Scope of the project \\
\hline $\mathrm{RI}^{112}$ & Business requirements \\
\hline $\mathrm{RI}^{113}$ & Readiness of the systems \\
\hline $\mathrm{RI}^{114}$ & Quality of the data \\
\hline $\mathrm{RI}^{115}$ & Implementation \\
\hline $\mathrm{RI}^{116}$ & Standardized result \\
\hline $\mathrm{RI}^{12}$ & PROGRAMMING \\
\hline $\mathrm{RI}^{121}$ & Dates \\
\hline $\mathrm{RI}^{122}$ & Estimate of time \\
\hline $\mathrm{RI}^{123}$ & Similar programming \\
\hline $\mathrm{RI}^{124}$ & Dependence \\
\hline $\mathrm{RI}^{13}$ & BUDGET \\
\hline $\mathrm{RI}^{131}$ & Elaboration \\
\hline $\mathrm{RI}^{132}$ & Financing \\
\hline $\mathrm{RI}^{14}$ & $\mathrm{RH}$ \\
\hline $\mathrm{RI}^{141}$ & The manager's experience \\
\hline $\mathrm{RI}^{142}$ & Experience of the team \\
\hline $\mathrm{RI}^{143}$ & Co-location \\
\hline $\mathrm{RI}^{144}$ & Backer of the project \\
\hline $\mathrm{RI}^{15}$ & $\mathrm{OTHER}$ \\
\hline $\mathrm{RI}^{151}$ & Requirements of the work \\
\hline $\mathrm{RI}^{152}$ & Impacts of the project \\
\hline $\mathrm{RI}^{153}$ & Affected departments \\
\hline $\mathrm{RI}^{154}$ & Participation will \\
\hline $\mathrm{RI}^{155}$ & Labor subjects \\
\hline & \\
\hline
\end{tabular}

\begin{tabular}{|c|c|}
\hline $\mathrm{RI}^{16}$ & TECHNOLOGY \\
\hline $\mathrm{RI}^{161}$ & Technological maturity \\
\hline $\mathrm{RI}^{162}$ & Technical requirements \\
\hline $\mathrm{RI}^{163}$ & Technological knowledge \\
\hline $\mathrm{RI}^{17}$ & PERFORMANCE \\
\hline $\mathrm{RI}^{171}$ & Performance objectives \\
\hline $\mathrm{RI}^{172}$ & Implementation easiness \\
\hline $\mathrm{RI}^{173}$ & Sub-contracts \\
\hline
\end{tabular}

\begin{tabular}{|c|c|}
\hline $\mathbf{R I}^{\mathbf{E}}$ & EXTERNAL FACTORS \\
\hline $\mathrm{RI}^{21}$ & FINANCIAL $\backslash$ ECONOMICAL \\
\hline $\mathrm{RI}^{211}$ & Inflation \\
\hline $\mathrm{RI}^{212}$ & Tax of exchange \\
\hline $\mathrm{RI}^{213}$ & Price of services \\
\hline $\mathrm{RI}^{214}$ & Stability Sub-contracts \\
\hline $\mathrm{RI}^{22}$ & POLITICAL \ENVIRONM. \\
\hline $\mathrm{RI}^{221}$ & Laws \\
\hline $\mathrm{RI}^{222}$ & Government \\
\hline $\mathrm{RI}^{223}$ & The community's position \\
\hline $\mathrm{RI}^{224}$ & Ambient \\
\hline $\mathrm{RI}^{225}$ & The community's interference \\
\hline $\mathrm{RI}^{23}$ & ACTIONS OF THE NATURE \\
\hline $\mathrm{RI}^{231}$ & Conditions of the time \\
\hline $\mathrm{RI}^{232}$ & Inundations \\
\hline $\mathrm{RI}^{233}$ & Windstorms \\
\hline $\mathrm{RI}^{234}$ & Fires \\
\hline $\mathrm{RI}^{235}$ & Earthquake \\
\hline
\end{tabular}

Table 2: $\quad$ Upper, medium and lower weights for each primary indicator.

\begin{tabular}{|l|c|c|c|}
\hline & $\mathrm{W}_{\mathrm{UB}}$ & $\mathrm{W}_{\mathrm{ME}}$ & $\mathrm{W}_{\mathrm{LB}}$ \\
\hline $\mathrm{RI}^{111}$ & 0,94 & 0,71 & 0,47 \\
\hline $\mathrm{RI}^{112}$ & 0,92 & 0,74 & 0,55 \\
\hline $\mathrm{RI}^{113}$ & 0,65 & 0,52 & 0,38 \\
\hline $\mathrm{RI}^{114}$ & 0,73 & 0,58 & 0,43 \\
\hline $\mathrm{RI}^{115}$ & 0,79 & 0,56 & 0,33 \\
\hline $\mathrm{RI}^{116}$ & 0,70 & 0,49 & 0,28 \\
\hline $\mathrm{RI}^{121}$ & 0,88 & 0,68 & 0,49 \\
\hline $\mathrm{RI}^{122}$ & 0,92 & 0,76 & 0,60 \\
\hline $\mathrm{RI}^{123}$ & 0,84 & 0,67 & 0,50 \\
\hline $\mathrm{RI}^{124}$ & 0,75 & 0,61 & 0,46 \\
\hline $\mathrm{RI}^{131}$ & 0,85 & 0,62 & 0,38 \\
\hline $\mathrm{RI}^{132}$ & 0,84 & 0,65 & 0,45 \\
\hline $\mathrm{RI}^{141}$ & 0,85 & 0,68 & 0,51 \\
\hline $\mathrm{RI}^{142}$ & 0,75 & 0,52 & 0,28 \\
\hline $\mathrm{RI}^{143}$ & 0,71 & 0,49 & 0,27 \\
\hline $\mathrm{RI}^{144}$ & 0,69 & 0,50 & 0,31 \\
\hline $\mathrm{RI}^{151}$ & 0,73 & 0,58 & 0,42 \\
\hline $\mathrm{RI}^{152}$ & 0,53 & 0,40 & 0,27 \\
\hline $\mathrm{RI}^{153}$ & 0,78 & 0,50 & 0,22 \\
\hline $\mathrm{RI}^{154}$ & 0,82 & 0,61 & 0,40 \\
\hline $\mathrm{RI}^{155}$ & 0,53 & 0,32 & 0,11 \\
\hline
\end{tabular}

\begin{tabular}{|l|c|c|c|}
\hline & W $_{\mathrm{UB}}$ & $\mathrm{W}_{\mathrm{ME}}$ & $\mathrm{W}_{\mathrm{LB}}$ \\
\hline $\mathrm{RI}^{161}$ & 0,78 & 0,55 & 0,32 \\
\hline $\mathrm{RI}^{162}$ & 0,80 & 0,61 & 0,41 \\
\hline $\mathrm{RI}^{163}$ & 0,76 & 0,58 & 0,41 \\
\hline $\mathrm{RI}^{171}$ & 0,84 & 0,63 & 0,41 \\
\hline $\mathrm{RI}^{172}$ & 0,87 & 0,68 & 0,48 \\
\hline $\mathrm{RI}^{173}$ & 0,85 & 0,62 & 0,39 \\
\hline $\mathrm{RI}^{211}$ & 0,58 & 0,37 & 0,17 \\
\hline $\mathrm{RI}^{212}$ & 0,82 & 0,61 & 0,40 \\
\hline $\mathrm{RI}^{213}$ & 0,66 & 0,50 & 0,34 \\
\hline $\mathrm{RI}^{214}$ & 0,66 & 0,45 & 0,25 \\
\hline $\mathrm{RI}^{221}$ & 0,59 & 0,37 & 0,16 \\
\hline $\mathrm{RI}^{222}$ & 0,49 & 0,31 & 0,13 \\
\hline $\mathrm{RI}^{223}$ & 0,28 & 0,18 & 0,08 \\
\hline $\mathrm{RI}^{224}$ & 0,39 & 0,24 & 0,08 \\
\hline $\mathrm{RI}^{225}$ & 0,25 & 0,18 & 0,11 \\
\hline $\mathrm{RI}^{231}$ & 0,40 & 0,25 & 0,11 \\
\hline $\mathrm{RI}^{232}$ & 0,27 & 0,16 & 0,06 \\
\hline $\mathrm{RI}^{233}$ & 0,43 & 0,29 & 0,15 \\
\hline $\mathrm{RI}^{234}$ & 0,35 & 0,22 & 0,09 \\
\hline $\mathrm{RI}^{235}$ & 0,30 & 0,15 & 0,01 \\
\hline
\end{tabular}

participant areas were: Weigh Control, Interiors, Environmental Systems, Landing Gear, Ground and Flight Test, Aeronautics, Propulsion, Configuration Engineering, Customer Support, RH and Planning. The Table 2 show the result of the weights below given by the specialists for each subject, compiled in upper, medium and lower limits. For the determination of the final values of risk (RI), 
the primary indicators were joined in the operational level, resulting later in new tactical and strategic levels indicators. For the analysis, it was chosen the use of the following linguistics terms: Low (trapezoidal), Medium (triangular) and High (trapezoidal). In other studies already observed were also used the following linguistics terms: Very low, Low, Medium, High and Very High - all in the triangular form. To arrive in this choice, simulations were accomplished with the two cases, where it was not obtained significant differences. This way, was opted for the simplest solution in the computational modelling vision and the specialist's understanding in the moment of the research. The final result, in agreement with the three notable points of the space of the alpha-cut approach solutions, is in Table 3:

Table 3: $\quad$ Results of the fuzzy analysis.

\begin{tabular}{|c|c|c|c|c|}
\hline & & $\mathbf{w}_{\mathrm{UB}} \cdot \mathbf{C}_{\mathrm{UB}}$ & $\mathbf{W}_{\mathrm{ME}} \cdot \mathbf{C}_{\mathrm{ME}}$ & $\mathbf{W}_{\mathrm{LB}} \cdot \mathbf{C}_{\mathrm{LB}}$ \\
\hline $\mathbf{R I}^{\prime}$ & INTERNAL FACTORS & 6,56 & 5,03 & 3,49 \\
\hline $\mathrm{RI}^{11}$ & MARK & 6,66 & 5,14 & 3,54 \\
\hline $\mathrm{RI}^{12}$ & PROGRAMMING & 8,59 & 6,12 & 4,36 \\
\hline $\mathrm{RI}^{13}$ & BUDGET & 5,75 & 4,60 & 2,58 \\
\hline $\mathrm{RI}^{14}$ & $\mathrm{RH}$ & 3,46 & 2,71 & 1,96 \\
\hline $\mathrm{RI}^{15}$ & OTHER & 6,05 & 4,61 & 2,96 \\
\hline $\mathrm{RI}^{16}$ & TECHNOLOGY & 5,71 & 3,89 & 1,15 \\
\hline $\mathrm{RI}^{17}$ & PERFORMANCE & 6,59 & 4,44 & 2,27 \\
\hline $\mathbf{R I}^{\mathrm{E}}$ & EXTERNAL FACTORS & 4,67 & 4,28 & 3,27 \\
\hline $\mathrm{RI}^{21}$ & FINANCIAL AND ECONOMICAL & 5,34 & 4,14 & 2,46 \\
\hline $\mathrm{RI}^{22}$ & POLITICAL AND ENVIRONMENTAL & 4,37 & 3,29 & 1,00 \\
\hline $\mathrm{RI}^{23}$ & ACTIONS OF THE NATURE & 3,86 & 3,41 & 1,19 \\
\hline $\mathbf{R I}$ & RISK INDICATOR & 5,59 & 4,68 & 4,10 \\
\hline
\end{tabular}

The final result demonstrates a medium risk for the project, which has already been proven thoroughly in the reality through other methodologies used for the risk control in the company of this case study. This final result, besides arriving in the same conclusion of other methodologies, still demonstrates a High Risk for the Programming item. This item also represented the actual problem, which, due to the short period for accomplishment of the development and certification of the appraised aircraft; had a delay in the programming when this study was performed.

\section{Conclusion}

This work joined two powerful analysis tools in a context of great complexity, which are the Risk management and the Fuzzy multicriteria analysis. The first, the approach of the risk in engineering projects, has been obtaining great relevance in the discussion of the subject of strategic administration at organizations and the second comes consolidating as a tool of multicriteria analysis. This union was expressed by the proposition of the system analysis in Figure 4 as strategic system of risk evaluation in engineering projects. Final results demonstrate a great reliability when compared to the tendency of the analyzed project. Moreover, other great advantages can be verified, such as: little need of specific data. Therefore it can be applied earlier (Preliminary phases of the project - profits in cost) and has the possibility of tracking the risk through the three levels of decision-making process: operational, tactical and strategic. 


\section{Reference}

[1] Project Management Institute (US) Standards Committee. A Guide to the Project management Body of Knowledge, PMI lnc.: Pennsylvania, 2004.

[2] Pender, S., Managing Incomplete Knowledge: Why Risk management is not Sufficient. International Journal of Project Management, 19, 79-87, 2001.

[3] Williams, T. M., The need for new paradigms for complex projects. International Journal of Project Management, Volume 17, 269-273, 1999.

[4] Kangari, R. \& Riggs L.S., Construction Risk assessment by Linguistics. IEEE transactions on Engineering Management, 36 (2), 126-131, 1989.

[5] Antonsson, E. K., Otto, K. N., Imprecision in Engineering Design. ASME Journal of Mechanical Design, 117(B), 1995.

[6] Ayyub, B. M., Karaszewski, Z. \& Klim, Z., Fuzzy-Based Decision Analysis for Risk Assessment of Marine Systems. AIAA Fórum, No 99 $1578,1999$.

[7] Mustafa, M.A., \& AI-Bahar, J.F., Project risk assessment Using the Analytic Hierarchy Process. IEEE transactions on Engineering Management, 38 (1), 46-52, 1991.

[8] Akintoye, A.S. \& MacLeod, M.J., Risk Analysis and Management in Construction. International Journal of Project Management, 15 (1), 1997.

[9] Kuchta, O., Use of Fuzzy Numbers in Project (Criticality) Assessment. International Journal of Project Management, 19, 305-310, 2001.

[10] Miller, R. \& Lessard, O., Understanding and Managing Risks in Large Engineering Projects. International Journal of Project Management, 19, 437-443, 2001.

[11] Floricel, S. \& Miller, R., Strategizing for Anticipated Risks and turbulence in Large-scale Engineering projects. International Journal of Project Management, 19, 445-455, 2001.

[12] Charoenngam, C. \& Yeh, C.Y., Contractual Risk and Liability Sharing in Hydropower Construction. International Journal of Project Management, 17 (1), 29-37, 1999.

[13] Zadeh, L.A., Outline of a New Approach to the Analysis of Complex Systems and Decision Processes. IEEE Transactions on Systems, Man and Cybernetics 3, 28-44, 1973.

[14] The MathWorks. Fuzzy Logic Toolbox User's Guide. The MathWorks, Inc.: Natick, 2002.

[15] Gheorghe, R., Bufardi, A., Xirouchakis, P., Construction of a twoparameters fuzzy outranking relation from fuzzy evaluation. Fuzzy Sets and Systems 143, 391-412, 2004.

[16] Gheorghe, R., Bufardi, A. \& Xirouchakis, P., Construction of global fuzzy preference structures from two-parameter single-criterion fuzzy outranking relations. Fuzzy Sets and Systems 153, 303-330, 2005.

[17] Dinsmore, P. C., \& Cavalieri, A. Como se tornar um profissional em gerenciamento de projetos. Qualitymark: Rio de Janeiro, 2003. 